\title{
Glassy state of native collagen fibril
}

\author{
S.G. Gevorkian ${ }^{1)}$, A.E. Allahverdyan ${ }^{1)}$, D.S. Gevorgyan ${ }^{2)}$, Chin-Kun Hu ${ }^{3)}$ \\ 1) Yerevan Physics Institute, Alikhanian Brothers St. 2, Yerevan 375036, Armenia. \\ ${ }^{2)}$ Yerevan State Medical University, Koryun St. 2, Yerevan, 375025, Armenia. \\ ${ }^{3)}$ Institute of Physics, Academia Sinica, Nankang, Taipei 11529, Taiwan.
}

\begin{abstract}
Our micromechanical experiments show that at physiological temperatures type I collagen fibril has several basic features of the glassy state. The transition out of this state [softening transition] essentially depends on the speed of heating $v$, e.g., for $v=1 \mathrm{C} / \mathrm{min}$ it occurs around $70 \mathrm{C}$ and is displayed by a peak of the internal friction and decreasing Young's modulus. The softening transition decreases by $45 \mathrm{C}$ upon decreasing the heating speed to $v=0.1 \mathrm{C} / \mathrm{min}$. For temperatures $20-30$ $\mathrm{C}$ the native collagen fibril demonstrates features of mechanical glassines at oscillation frequencies 0.1-3 kHz; in particular, the internal friction has a sharp maximum as a function of the frequency. This is the first example of biopolymer glassines at physiological temperatures, because well-known glassy features of DNA and globular proteins are seen only for much lower temperatures (around $200 \mathrm{~K})$.

PACS numbers: 36.20.-r, 36.20.Ey
\end{abstract}

An important aspect of the globular protein physics is the low-temperature glass transition experimentally observed at $\approx 200 \mathrm{~K}[1-8]$. The understanding of this transition is achieved by means of combining the information obtained via different methods: micro-mechanical experiments [1], NMR [2], Moessbauer spectroscopy [3], calorimetric studies [4], pressure release experiments [5], and X-ray scattering of synchrotron radiation [6]; see [7, 8] for reviews. Although there still open questions here - concerning, in particular, the specific role of the hydrated water - the rough physical picture of the glass transition in globular proteins is constructed by an analogy with glass-forming liquids [7, [8] and synthetic polymers $[9,10]$. In particular, it is believed that the largescale conformational motion of proteins freezes at $\approx 200$ $\mathrm{K}$, analogously to freezing of cooperative motion in glassforming liquids [7, 8] and segmental motion in synthetic polymers [9, 10].

Thus, the glassy features as such are not important in the native state of globular proteins at physiological temperatures, though the glass transition at much lower temperatures allows to gain some understanding of relevant motions in proteins $[7,8]$.

Here we shall demonstrate via micro-mechanical methods that the native type I collagen fibril (made of fibrous protein, type I collagen triple-helices) is in a glassy state at physiological temperatures. This state is displayed via frequency-dependent visco-elastic characteristics (the Young's modulus and the damping decrement) of the native fibril. Upon heating the fibril goes out of the glassy state, a phenomenon known as the softening transition [9, 10]. The temperature of this transition depends essentially on the speed of heating, e.g., for the standard heating speed $v=1 \mathrm{C} / \mathrm{min}$ it occurs around $\approx 70 \mathrm{C}$. Note that around $70 \mathrm{C}$ the fibril starts to undergo the denaturation process [13 16]. This process has been studied by different methods, and some of those methods seems to

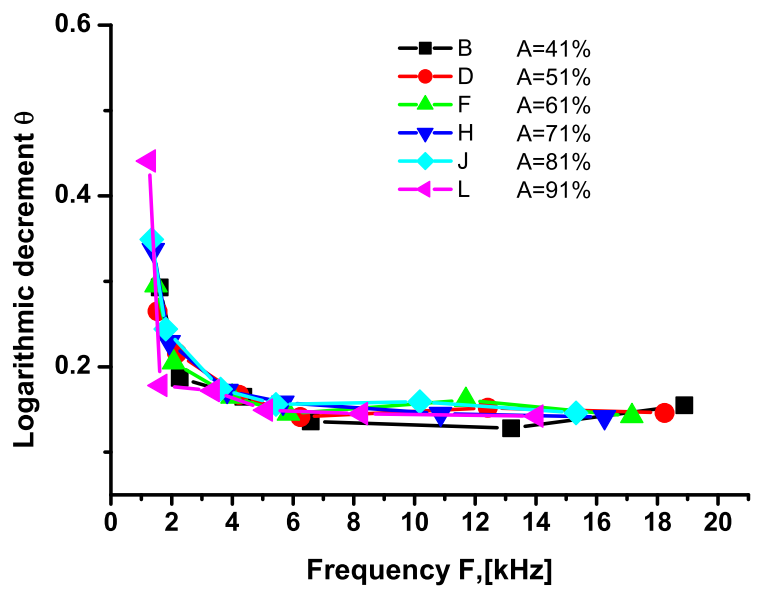

FIG. 1: The logarithmic damping decrement $\theta$ of the collagen fibril versus the frequency at temperature $25^{\circ} \mathrm{C}$ and varying humidity.

indicate that this process is inherently irreversible [14]. We saw that upon decreasing the heating speed to $v=0.1$ $\mathrm{C} / \mathrm{min}$, the softening transition takes place at $\approx 25 \mathrm{C}$, showing that the glassy features pertain to the native state of the collagen fibril and do not directly relate to its denaturation. We shall also confirm that the glassy features are not seen for the heat-denaturated collagen fibril, and contrast features of the native collagen fibril to those of globular proteins.

Type I collagen is the major structural element in the extra-cellular matrix. It forms the basis of fibrous connective tissues, such as tendon, chord, skin, bones, cornea and dentine; see [11, 12] for recent reviews. Collagen ensures the mechanical stability and strength of these tissues, but its biological role is certainly larger because it 


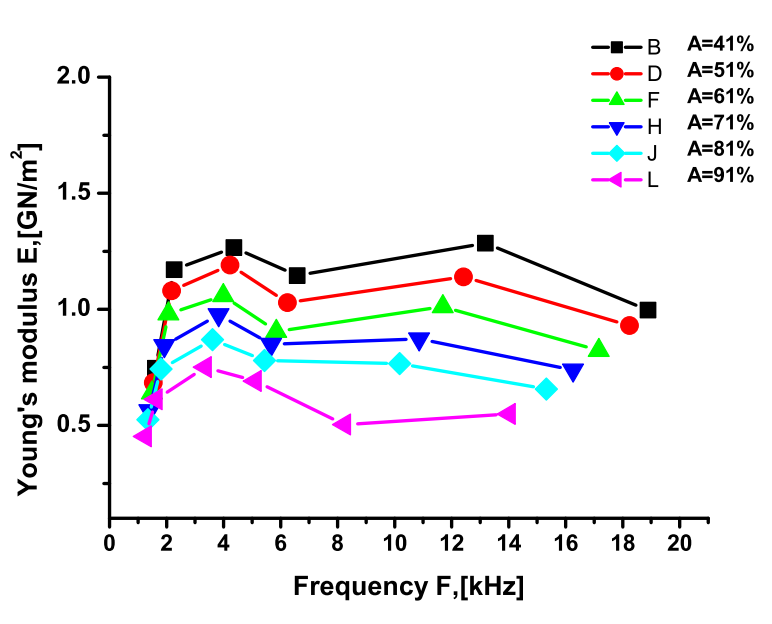

FIG. 2: The Young's modulus $E$ of the collagen fibril versus the frequency at temperature $25^{\circ} \mathrm{C}$ and varying humidity.

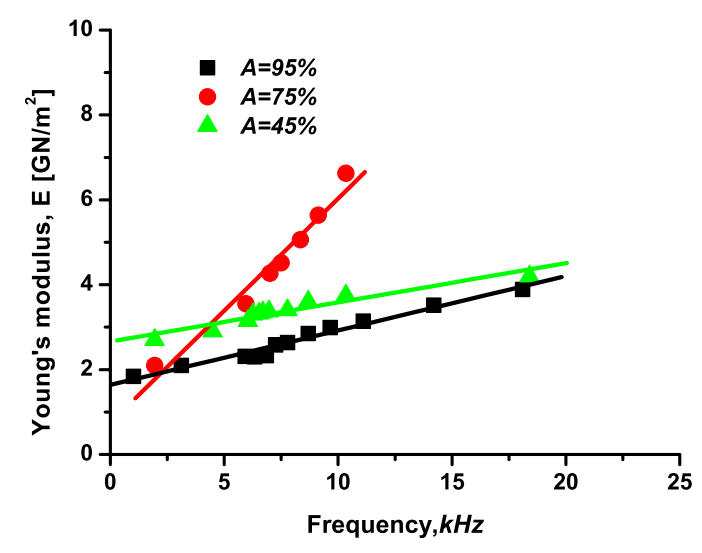

FIG. 3: The Young modulus $E$ versus frequency of lysozyme crystal for $T=25 \mathrm{C}$ and varying humidity $A$.

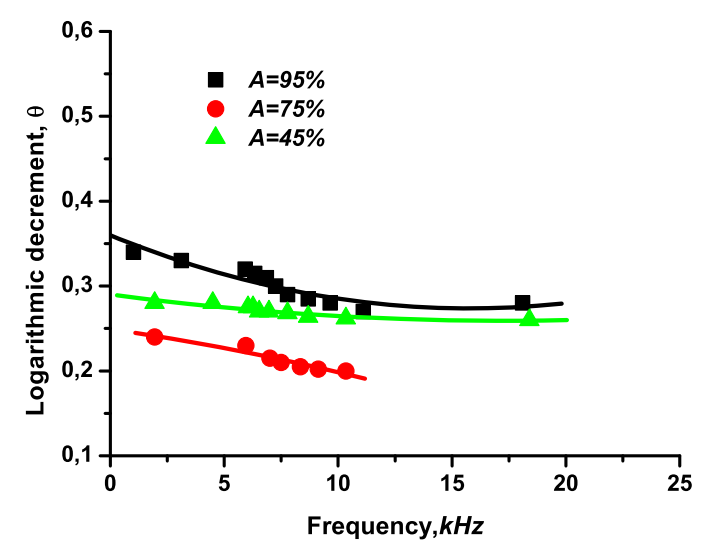

FIG. 4: The logarithmic damping decrement of lysozyme crystal versus frequency for $T=25 \mathrm{C}$ and varying humidity $A$. also participates in biochemical and immunological protection of the organism. Connective tissues are macroscopic hierarchical structures that consist of several levels: a collagen tendon is made of several fascicles held together. The fascicle consists of fibers, which are made (going to the bottom of the hierarchy) from fibrils, microfibrils and collagen triple-helices (which can be of several types, e.g., type I) [11]. Each triple-helix consists of three poly-peptide chains wound around each other. Fibrils and microfibrils are stabilized by several different factors: a small number of covalent links between terminal points of collagen triple-helices, carbonyl-water hydrogen-bonds, hydrophobic and van der Waals interaction between triple-helices [11, 12].

Materials and Methods. Collagen fibril samples with diameter from $1 \mu \mathrm{m}$ and the length $1-0.1 \mathrm{~mm}$ were extracted from Achilles tendons of young rats by means of shaking and pulling using micro-tweezers in $96 \%$ of ethyl alcohol at temperature $5 \mathrm{C}$. We call our sample fibril, because its diameter is closer to the accepted diameter of the fibril than to that of the fiber. Indeed, the fibril diameter established via electron microscopy varies between $40 \mathrm{~nm}$ and $0.5 \mu \mathrm{m}$ [11]. For real fibrils this value is underestimated as the electron microscopy demands drying of the collagen samples.

The sample under investigation was enclosed in the experimental chamber and placed in a temperaturecontrolled cabinet with the temperature maintained at $25^{\circ} \mathrm{C}$. The sample was allowed to equilibrate at a given humidity for several hours. The relative humidities from 97 to $32 \%$ in the chamber were achieved by means of $\mathrm{CaCl}_{2}$ solutions of different concentrations, while the relative humidities of $15 \%$ and $10 \%$ were obtained via saturated solutions of $\mathrm{ZnCl}_{2}$ and $\mathrm{LiCl}$, respectively.

The Young's modulus $E$ and the logarithmic damping decrement $\theta$ were measured via electrically excited transverse resonance vibrations of the sample (fibril cylinder), which is cantilevered from one edge (another edge is free) 1]. $E$ characterizes the elasticity and defined as the ratio of pressure over strain [9, 10]; $\theta$ is determined from the amplitude of the sample oscillations and is defined as the ratio of the energy dissipated during the externally forced oscillations to the energy (reversibly) stored in the sample 9,10$]$. A large $\theta$ is typical for viscous liquids, while a small $\theta$ characterizes elastic solids.

For measuring $E$ and the amplitude-frequency characteristics of oscillations (employed for obtaining $\theta$ ), it is necessary to change smoothly the frequency $f$ of the induced oscillations and determine the resonance frequency, which corresponds to the maximal oscillation amplitude of the sample's free end. The Young's modulus of sample's main axis is calculated via [17]

$$
E=3.19 \cdot \omega^{2} \cdot L^{4} \cdot \rho \cdot P / I_{\min },
$$

where $\omega$ is the resonance frequency, $L$ is the sample length, $P$ is the cross-section area, $\rho$ is the density, and 


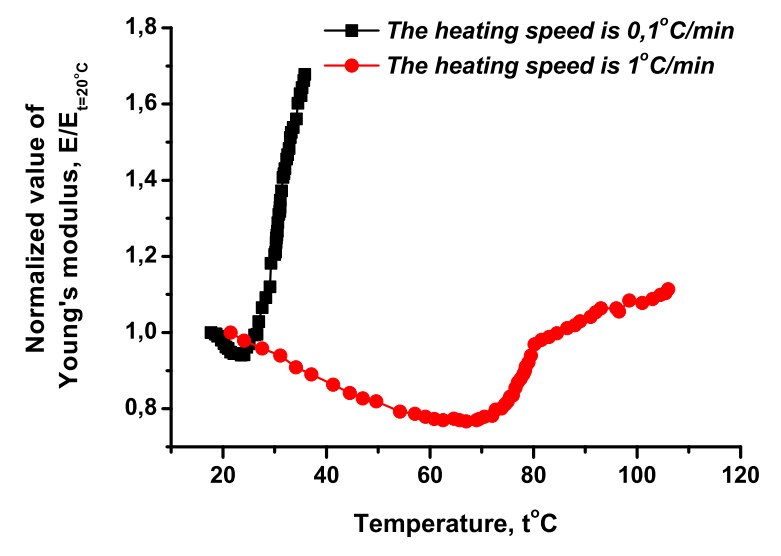

FIG. 5: The normalized Young's modulus $E$ of the collagen fibril versus temperature for different heating speeds. The heating starts from $19 \mathrm{C}$ and relative humidity $A=93 \%$.

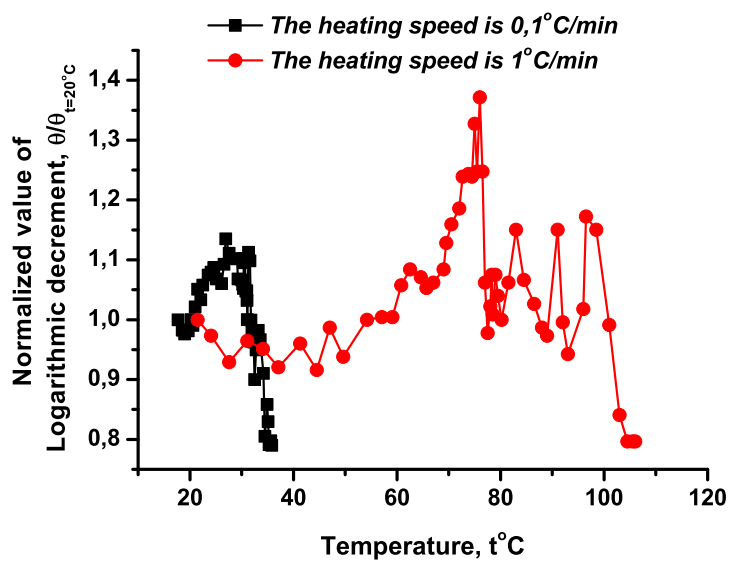

FIG. 6: The normalized logarithmic damping decrement $\theta$ of the collagen fibril versus temperature for different heating speeds. The heating starts from $19 \mathrm{C}$ and $A=93 \%$.

$I_{\min }$ is the main inertia moment of that section, which corresponds to the deformation plane with the minimal stiffness. For the round cross-section of our samples $I_{\min }=\pi \cdot D^{4} / 64$ [17] and $P=\pi \cdot D^{2} / 4$, where $D$ is the sample diameter (measured with precision $0.02 \mu \mathrm{m}$ ). Thus the Young's modulus is obtained from (11), where $L, \rho, P$ and $I_{\min }$ are the known sample parameters and $\omega$ is measured on the experiment. Studying samples with different length $L$, one can explore a range of frequencies.

Below we contrast the native features of collagen fibril with those of globular protein lysozyme (in solid state). Tetragonal $\left(P 4_{3} 2_{1} 2\right)$ lysozyme crystals were grown following the method of [19] from a solution containing $3 \%$ of lysozyme, $5 \%$ of $\mathrm{NaCl}, 0.2 \mathrm{M}$ of natrium-acetate buffer, and $\mathrm{pH}=4.7$. Crystals were fixated for 10 days via diffusion through the gas phase of glutaraldehyde. For another 10 days the crystalls were fixated directly into $5 \%$ glutaraldehyde solution with the same buffer. Fixated crystalls have parameters of the elementary units $(a=b=79.4 \mathrm{~A}$ and $c=37.6 \mathrm{~A})$ close to native ones: $a=b=79.1 \mathrm{~A}$ and $c=37.9 \mathrm{~A}[20$.

Mechanical glassy state. Glass is a meta-stable state of matter, which is caught in the process of (very) slow relaxation to equilibrium [9, 10]. Features of this state are sensitive to observation times (frequencies). It is typically displayed whenever the system does not have enough time to follow the external changes, e.g., when cooling with a small, but finite rate, or forcing the system with a finite frequency. These two types of glassines are known, respectively, as structural and mechanical [9, 10].

During the mechanical glass transition the system is forced by an external field at a frequency $\omega$ (with us this is the resonance frequency of the sample vibration). When changing $\omega$ at a fixed temperature $T$ (this is achieved by changing the sample length), the damping decrement $\theta(\omega)$ - which characterizes internal friction and thus energy dissipation - displays a maximum at some frequency $\omega_{\mathrm{g}}[9,10]$. Approximately at the same frequency range $\omega \approx \omega_{\mathrm{g}}$, the Young's modulus $E(\omega)$ (or some other elastic modulus) displays a crossover between a relatively large high-frequency value $E_{+}$to a relatively small, low-frequency value $E_{-}[9,10]$. At the glass transition frequency $\omega_{\mathrm{g}}$ (one of) the relaxation time(s) of the systems becomes of order of $\left.1 / \omega_{\mathrm{g}}, 9,10\right]$. In structural glass-formers the maximum of internal friction corresponds to a large viscosity, while the two values $E_{+}$ and $E_{-}$of $E$ refer respectively to solid-like and liquidlike elaticity features. The same behavior of $E$ and $\theta$ is seen for a fixed frequency $\omega$ and the temperature changing at a small, but finite speed. The glass transition now refers to the relaxation time being of the same order as the inverse dimensionless temperature speed [9, 10].

Results and discussion. Fig. 1 displays the damping decrement $\theta$ of the collagen fibril versus frequency $\omega$ and at varying humidity and room temperature $25 \mathrm{C}$. Recall that this is the resonance frequency of the sample at which we measure the Young's modulus; we remind as well that the frequency varies with the sample length. It is seen from Fig. 1 that $\theta$ has a well-displayed maximum at frequencies lower than $2 \mathrm{kHz}$ and that the behavior of $\theta$ does not depend on the humidity. The plateau behavior of the Young's modulus $E$ at the same temperature $25 \mathrm{C}$ is seen on Fig. 2, The plateau is most clealry visible at lowest studied humidity, though it is identifiable for the highest humidity as well; see Fig. 2.

The results presented on Figs. 1 and 2 imply that the room temperature collagen fibril displays glassy features at room temperature. The transition frequency $\omega_{\mathrm{g}}$ is smaller than $2 \mathrm{kHz}$ at $T=25 \mathrm{C}$. Changing the humidity does not alter $\omega_{\mathrm{g}}$, in contrast to the low-temperature glassy features of globular proteins, where the hydration level is known to be essential [7, 8]. For the collagen fibril only the upper value $E_{+}$of the Young's modulus 
decreases (the fibril becomes more flexible) for a higher humidity. To make the comparison with globular proteins even more visible we studied visco-elastic features of crystalline lysozyme at room temperature (each site of the tetragonal crystal contains one globular lysozyme protein). Results presented in Figs. 3, 4, show no indications of glassy behavior. It is also seen that for the native lysozyme the dependence on the humidity is essential.

It is expected that upon heating the fibril will go out of the glassy state. For synthetic polymers this phenomenon is known as the softening transition [9, 10]; this transition is accompanied by the same effects as the glass transition: a peak of the internal friction and a relatively abrupt change of the Young's modulus. Figs. 5], 6 show that when heating at speed $1 \mathrm{C} / \mathrm{min}$ the collagen fibril undergoes softening transition at temperatures around $T_{\text {soft }}=70 \mathrm{C}$, where $\theta$ has a sharp maximum. We note however that the behavior of the Young's modulus $E$ is more intricate [18] than during the glass transitions in synthetic polymers, e.g., rosin [9, 10]. It is seen that for $T<T_{\text {soft }}, E$ decreases upon increasing $T$; this is a typical scenario for the softening transition. However, for $T_{\text {soft }} \lesssim T$ the Young's modulus starts to increase with temperature. This effect was first found in Ref. [18]; it was argued to be related to formation of inter-molecular bonds between partially denaturated fibril constituents. Note that the denaturation temperature of collagen tendon, as measured calorimetrically, is also located around $60-70 \mathrm{C}$ when heating under speed larger than $1 \mathrm{C} / \mathrm{min}[13-16$. Thus, around $70 \mathrm{C}$ there are three diferent processes taking place almost simultaneously: partial denaturation of fibril, formation of inter-molecular bonds which increases the Young's modulus for higher temperatures, and the softening transition. Altogether, the collagen physics around $70 \mathrm{C}$ is complicated. Now upon decreasing the heating speed the softening transition should decrease, because for slower heating a lower temperature suffice for displaying the relevant motion within the observation times [10]. This is what we saw in our experiments: for the heating speed $0.1 \mathrm{C} / \mathrm{min}$ we get $T_{\text {soft }} \approx 25 \mathrm{C}$; see Figs. 5. 6. This is the physiological temperature regime for the native collagen. It is to be stressed that the glassy features - including frequency-dependences and the softening transition - are absent for the heat-denaturated collagen fibril, which is prepared by keeping the native sample at $120 \mathrm{C}$ for several hours. Thus the ordered character of the fibril is essential for displaying the glassy features. We conjecture that these features are built in during the fibrilogenesis; they can be similar to the orientational glassines phenomenon known for synthetic polymers [10].

In this context it is interesting to note that the calorimetric studies carried out in Refs. [14, 16] did indicate on the presence of certain glassy feature in the heatdenaturated collagen tendon. It was also seen that these features are very sensitive to hydration changes, and that they tend to disappear after annealing the heatdenaturated collagen tendons [16]. There are here two essential differences with respect to our results. First, we found glassy features in the native (and not heatdenaturated) state of collagen fibril. Second, the hydration level is not essential for these features, e.g., Figs. 1, 2 shows that changing the humidity level is not essential for the characteristic glassy frequency $\omega_{\mathrm{g}}$.

We close by repeating the main message of the present work: type I collagen fibril is glassy at its physiological temperatures. The phenomenon is not sensitive to hydration changes, is specific to the native state of the fibril, and constitutes the first example of biopolymer glassiness at physiological temperatures. It may be of relevance for understanding the genesis of collagen-based structures and for controlling their aging.

This work was supported by Volkswagenstiftung, ANSEF, SCS of Armenia (grant 08-0166), and grants 962911-M 001-003-MY3 \& AS-95-TP-A07 of NSC-Taiwan.

[1] V.N. Morozov and S.G. Gevorkian, Biopolymers, 24, 1785 (1985).

[2] A. G. Palmer III, J. Williams and A. McDermott, Phys. Chem. 100, 13293 (1996).

[3] E. W. Knapp et al., J. Chem. Phys. 78, 4701 (1983).

[4] G. Sartor, E. Mayer and G. P. Johari, Biophys. J., 66, 249, (1994).

[5] I.E.T. Iben et al., Phys. Rev. Lett. 62, 1916 (1989).

[6] K. Achterhold et al., Eur. Biophys. J., 25, 43 (1996). C. Keppler et al., Eur. Biophys. J., 25, 221 (1997).

[7] H. Frauenfelder et al., Science 254, 1598 (1991).

[8] D. Ringe and G. A. Petsko, Biophysical Chemistry 105, 667 (2003).

[9] J. D. Ferry, Viscoelastic Properties of Polymers (John Wiley \& Sons, New York, 1980).

[10] G. M. Bartenev and S. Ya. Frenkel, Physics of Polymers (Khimia, Leningrad, 1990) (In Russian).

[11] Collagen, Structure and Mechanics, ed. by P. Fratzl (Springer, Berlin, 2008).

[12] J. Engel and H. P. Bachinger, Top. Curr. Chem. 247, 7 (2005).

[13] A. R. Haly and J. W. Snaith, Biopolymers, 10, 1681 (1971). A. Finch and D. A. Ledward, Biochim. Biophys. Acta, 278, 433 (1972).

[14] C. A. Miles, T. V. Burjanadze and A. J. Bailey, J. Mol. Biol. 245, 437 (1995). C. A. Miles and A. J. Bailey, Proc. Indian Acad. Sci. (Chem. Sci.), 111, 71 (1999).

[15] E. I. Tiktopulo and A. V. Kajava, Biochemistry, 37, 8147 (1998).

[16] G. I. Tsereteli et al., Journal of Thermal Analysis and Calorimetry, 62, 89 (2000).

[17] L.D. Landau and E.M. Lifshitz, Elasticity Theory (Nauka Publishers, Moscow, 1965).

[18] S. G. Gevorkian et al., Phys. Rev. Lett. 102, 048101 (2009).

[19] L. K. Steinrauf, Acta Crystallogr. 12, 77 (1959).

[20] C.C.P. Blake et al. Nature, 206, 757 (1965). 